\title{
ISOPERIMETRIC INEQUALITIES AND IMBEDDING THEOREMS IN IRREGULAR DOMAINS
}

\author{
PIOTR HAJŁASZ AND PEKKA KOSKELA
}

\begin{abstract}
The paper proves several weighted imbedding theorems for domains with fractal boundaries. The weights considered are distances to the boundary to certain powers, and the domains are so-called $s$-John domains. The paper also proves, in the general setting, that the existence of an imbedding implies compactness of the imbedding for lower exponents. Moreover, following Maz'ya, the paper reformulates the imbedding theorems in the language of local isoperimetric and capacity estimates.
\end{abstract}

\section{Introduction}

The Sobolev-Poincaré inequality

$$
\left(\int_{\Omega}\left|u-u_{\Omega}\right|^{n p /(n-p)} d x\right)^{(n-p) / n p} \leqslant C\left(\int_{\Omega}|\nabla u|^{p} d x\right)^{1 / p}
$$

holds for $1 \leqslant p<n$ whenever $u$ is smooth, $u_{\Omega}=f_{\Omega} u d x=|\Omega|^{-1} \int_{\Omega} u d x$, and $\Omega$ is sufficiently nice, that is, say, that it is a bounded domain and satisfies the cone condition. In the case in which $1<p<n$, this result is due to Sobolev [43, 44]. The case in which $p=1$ was a long standing open problem until elementary proofs were given by Gagliardo [15] and Nirenberg [39]. They also showed that the inequality for $1<p<n$ can be easily deduced from that for $p=1$ (cf. [51, Theorem 2.4.1]). The next progress was made independently by Federer and Fleming [13] and by Maz'ya [28].

If $p=1$ and $\Omega=\mathbb{R}^{n}$, then we can put $u_{\Omega} \equiv 0$ in (1):

$$
\left(\int_{\mathbb{R}^{n}}|u|^{n /(n-1)} d x\right)^{(n-1) / n} \leqslant C(n) \int_{\mathbb{R}^{n}}|\nabla u| d x .
$$

Federer and Fleming [13] established this inequality with the best possible constant $C(n)$ by using the co-area formula and by applying the isoperimetric inequality to the level sets of $u$. This proof shows that, in a certain sense, inequality (2) is equivalent to the isoperimetric inequality (cf. [51, Theorem 2.7.4, Remark 2.7.5]). Maz'ya [28] verified a stronger result: if $\Omega \subset \mathbb{R}^{n}$ is an arbitrary open set with finite volume, and $1 \leqslant q \leqslant n /(n-1)$, then the inequality

$$
\left(\int_{\Omega}\left|u-u_{\Omega}\right|^{q} d x\right)^{1 / q} \leqslant C \int_{\Omega}|\nabla u| d x
$$

holds if and only if a version of the isoperimetric inequality involving exponent $q$ is

Received 5 June 1995; revised 10 March 1996.

1991 Mathematics Subject Classification 46E35.

The first author was partially supported by KBN grant 2-PO3A-034-08, and the second author by the Academy of Finland and by the NSF.

J. London Math. Soc. (2) 58 (1998) 425-450 
satisfied by the subsets of $\Omega$ (cf. [33, Corollary 3.2.3]). As a consequence, he also obtained inequality (2) with the best constant.

The equivalence of the Sobolev imbedding of the type of inequality (3) with the isoperimetric inequality also has counterparts in the theory of finitely generated groups and graph theory, as well as in the theory of Markov operators [26, 49, 50]. Roughly speaking, in the terminology of Gromov, the proof of inequality (3) and its counterparts in the abstract settings of graphs and Markov operators is reduced to the computation of the 'isoperimetric dimension' of the space.

As already mentioned, inequality (1) with $1<p<n$ follows easily from that for $p=1$. Moreover, if $|\Omega|<\infty$, and if inequality (3) holds for a fixed $1 \leqslant q \leqslant n /(n-1)$ and all $u \in C^{\infty}(\Omega)$, then the same method (cf. the proof of Corollary 3 ) leads to

$$
\left(\int_{\Omega}\left|u-u_{\Omega}\right|^{s} d x\right)^{1 / s} \leqslant C\left(\int_{\Omega}|\nabla u|^{p} d x\right)^{1 / p}
$$

where $p<q /(q-1), s=p q /(p+q-p q)$. In passing from (3) to (4), one does not need to impose any regularity assumptions on $\Omega$. In the case of a bounded domain with the cone property, $q=n /(n-1)$, and hence we get the exponent $s=n p /(n-p)$ in (4), which is the best possible. However, it was observed by Maz'ya [33] that for more complicated domains this method does not necessarily yield the best possible exponent in (4). To obtain the best exponent in (4) for $p>1$, one has to deal with 'capacity estimates' for level sets rather than with isoperimetric inequalities. Maz'ya $[28-30,32]$ proved that inequality (4) holds if and only if certain capacity estimates involving exponents $s$ and $p$ are satisfied by the subsets of $\Omega$. This result extends to the general setting of Markov operators [26].

For a full treatment of the above mentioned necessary and sufficient conditions for the existence of various kinds of imbedding theorem in open subsets of $\mathbb{R}^{n}$, we refer the reader to Maz'ya's monograph [33, Chapters 3 and 4], and to Section 2 of this paper. However, this abstract approach does not solve the entire problem, because in general it is very difficult to check whether subsets of a given domain satisfy these isoperimetric or capacity estimates. The most general class of domains for which Maz'ya [33] verifies such estimates is the class of bounded domains with the cone condition. Apart from this class, he only deals with particular examples.

Imbedding theorems for more general classes of domain were later established with different methods. Bojarski [3] proved inequality (1) when $1 \leqslant p<n$ for a very general class of 1-John domains, using the chaining method of Boman [4]. This method of proof and certain variants of it were then also employed by Chua [10], Hurri [20-22], and Smith and Stegenga [41, 42] and others.

In this paper we present a rather elementary method for establishing these isoperimetric and capacity estimates for a large class of domains. In particular, we verify weighted inequalities in $s$-John domains (see Section 3 for the definition) and unify different approaches. Our results cover and extend some of the earlier results of Bojarski [3], Hurri [20-22], Smith and Stegenga [41, 42], and people from the Czech school (see [40], and references therein). Our interest in $s$-John domains in part arises from a recent paper by Buckley and the second author of this paper [6], which shows that all simply connected plane domains that satisfy a Sobolev-Poincaré type inequality are $s$-John for an appropriate $s$ (for inequality (1), $s=1$ ).

Also, we prove a very general version of the Rellich-Kondrachov compactness theorem that we have not been able to locate in the literature. Namely, we prove that, 
in the general setting of weighted spaces, the existence of the imbedding $W^{1, p} \subset L^{q}$ implies that, for all $s<q$, the imbedding $W^{1, p} \subset L^{s}$ is compact, provided that the weighted measure of the domain is finite (Theorem 5). This observation has a very simple proof, and it immediately reduces the question of compactness to the existence of an imbedding, a fact that seems to have been overlooked by a number of authors. The unweighted case with $p=1$ of our conclusion can be found in a bit implicit form in Maz'ya's work [28; 33, Corollary 4.8.3.3].

The paper is organised as follows. In Section 2, we prove a general result (Theorem 1) in the spirit of Maz'ya [33]. This result states that, in a given domain, a weighted imbedding holds if and only if subsets of this domain satisfy certain weighted isoperimetric or weighted capacity estimates. In the unweighted case, this result is essentially due to Maz'ya. Related weighted variants were considered by Maz'ya $[\mathbf{3 1} ; 33$, pp. 112, 113] and Stredulinsky [46]. Our approach seems to be slightly more elementary than that of Maz'ya and Stredulinsky (see [33, Chapters 3 and 4; 46, Chapter 2]). In Section 2, we also prove the compactness theorem referred to above.

In Section 3, we recall the definitions and some basic properties of the $s$-John domains, $1 \leqslant s<\infty$. These domains can have very wild boundaries.

In Section 4, we establish weighted isoperimetric and weighted capacity estimates for subsets of $s$-John domains. Thus, applying Theorem 1 from Section 2, we obtain a general imbedding theorem in $s$-John domains. Compactness of imbedding is then a corollary from the general compactness result in Section 2. We also introduce an 'envelope extension operator', and prove the existence of such an operator for 1-John domains (Theorem 10).

The symbol $\Omega$ will always denote an open subset of $\mathbb{R}^{n}$, where $n \geqslant 2$. By $\chi_{E}$, we denote the characteristic function of the set $E$. The average value of $u$ with respect to the measure $\mu$ is denoted by $u_{\Omega, \mu}=f_{\Omega} u d \mu=\mu(\Omega)^{-1} \int_{\Omega} u d \mu$. If $d \mu(x)=f(x) d x$ with $0<f \in L_{\mathrm{loc}}^{1}(\Omega)$, then we write $u_{\Omega, f}$ instead of $u_{\Omega, \mu}$. In the case in which $\mu$ is the Lebesgue measure, we simply write $u_{\Omega}$. By $L^{p}(\Omega, f)$, we denote the $L^{p}$ space with respect to the measure $\mu$ given by $d \mu(x)=f(x) d x$. By $H^{k}$, we denote the $k$-dimensional Hausdorff measure.

By $C$, we denote a general positive constant. This can change its value even in a single line. By writing $C=C(p, q, \lambda)$, we indicate that the constant $C$ depends on $p$, $q$ and $\lambda$ only. We write $u \approx v$ to state that there exist two positive constants $C_{1}$ and $C_{2}$ such that $C_{1} u \leqslant v \leqslant C_{2} u$.

\section{Reformulation of the imbedding theorems}

In this section we consider weighted inequalities. The weights are generated by positive and continuous functions. Let us begin with the following elementary technical observation.

LEMMA 1. Let $f$ and $g$ be two continuous and positive functions on an open set $\Omega \subset \mathbb{R}^{n}$. Assume that $\int_{\Omega} f(x) d x<\infty$. Let $1 \leqslant p \leqslant q<\infty$. Then the following three conditions are equivalent:

(1) There exists a constant $C>0$ such that, for every $u \in C^{\infty}(\Omega)$,

$$
\inf _{c \in \mathbb{R}}\left(\int_{\Omega}|u(x)-c|^{q} f(x) d x\right)^{1 / q} \leqslant C\left(\int_{\Omega}|\nabla u(x)|^{p} g(x) d x\right)^{1 / p} .
$$


(2) There exists a constant $C>0$ such that, for every $u \in C^{\infty}(\Omega)$,

$$
\left(\int_{\Omega}\left|u(x)-u_{\Omega, f}\right|^{q} f(x) d x\right)^{1 / q} \leqslant C\left(\int_{\Omega}|\nabla u(x)|^{p} g(x) d x\right)^{1 / p} .
$$

(3) For an arbitrary ball $B \subset \subset \Omega$, there exists a constant $C>0$ such that

$$
\left(\int_{\Omega}|u(x)|^{q} f(x) d x\right)^{1 / q} \leqslant C\left(\int_{\Omega}|\nabla u(x)|^{p} g(x) d x\right)^{1 / p}
$$

whenever $u \in C^{\infty}(\Omega)$ satisfies $\left.u\right|_{B}=0$.

Remark 1. Note that each of the above conditions implies that the set $\Omega$ is connected. Indeed, a function that is equal to 1 on one of the components and 0 on the remaining components would not satisfy the indicated inequality.

REMARK 2. Note that, if $\int_{\Omega} f(x) d x=\infty$, then condition (2) makes no sense, as the average $u_{\Omega, f}$ is not defined for, say, $u=1$. Moreover, condition (3) implies that $\int_{\Omega} f(x) d x<\infty$. Indeed, it suffices to apply condition (3) to a smooth function $u$ that is equal to 1 outside the ball $2 B \subset \subset \Omega$ and vanishes on $B$. Hence in the case $\int_{\Omega} f(x)=\infty$ the correct variant of the imbedding is condition (1). However, in this paper we shall only consider the case when the function $f$ is summable.

REMARK 3. Weighted Sobolev inequalities with weights $f$ and $g$ given by powers of the distance to the boundary are sometimes called Hardy inequalities.

REMARK 4. For one more equivalent statement, see Corollary 2.

Proof of Lemma 1. For simplicity, we shall use the notation $d \mu=f(x) d x$. Thus $u_{\Omega, f}=u_{\Omega, \mu}$. The equivalence of the first two statements follows from the elementary inequality

$$
\left(\int_{\Omega}\left|u-u_{\Omega, \mu}\right|^{q} d \mu\right)^{1 / q} \leqslant 2 \inf _{c \in \mathbb{R}}\left(\int_{\Omega}|u-c|^{q} d \mu\right)^{1 / q}
$$

Now we prove that condition (2) $\Rightarrow$ condition (3). Assume that $u \in C^{\infty}(\Omega)$ and $\left.u\right|_{B}=0$. We have

$$
\left(f_{\Omega}|u|^{q} d \mu\right)^{1 / q} \leqslant\left(f_{\Omega}\left|u-u_{\Omega, \mu}\right|^{q} d \mu\right)^{1 / q}+\left|u_{\Omega, \mu}\right|
$$

It suffices to estimate $\left|u_{\Omega, \mu}\right|$ :

$$
\left|u_{\Omega, \mu}\right|=\left|u_{\Omega, \mu}-u_{B, \mu}\right| \leqslant \oint_{B}\left|u-u_{\Omega, \mu}\right| d \mu \leqslant \frac{\mu(\Omega)}{\mu(B)}\left(\int_{\Omega}\left|u-u_{\Omega, \mu}\right|^{q} d \mu\right)^{1 / q} .
$$

The proof that condition (3) $\Rightarrow$ condition (1) is slightly more complicated. Since the functions $f$ and $g$ are continuous and positive, the measures generated by $f(x) d x$ and 
$g(x) d x$ are uniformly equivalent with the Lebesgue measure on every compact subset of $\Omega$. Also, the exponent $q$ in condition (3) cannot exceed that from the classical Sobolev inequality. Hence it easily follows that, for every $2 B \subset \subset \Omega$, the inequality

$$
\left(\int_{2 B}|u(x)|^{q} f(x) d x\right)^{1 / q} \leqslant C\left(\int_{2 B}|\nabla u(x)|^{p} g(x) d x\right)^{1 / p}
$$

holds for all $u \in C_{0}^{\infty}(2 B)$ with $C$ depending on $B, f$ and $g$. Moreover, the Poincaré inequality

$$
\left(\int_{2 B \backslash B}\left|u-u_{2 B \backslash B}\right|^{p} g(x) d x\right)^{1 / p} \leqslant C\left(\int_{2 B \backslash B}|\nabla u|^{p} g(x) d x\right)^{1 / p}
$$

holds for all $u \in C^{\infty}(2 B \backslash B)$, with $C$ depending on $B$ and $g$. Let $\in C_{0}^{\infty}(2 B)$ satisfy $\left.\right|_{B}=1$. Now Lemma 1 follows when condition (3) and inequalities (5) and (6) are applied to

$$
u-u_{2 B \backslash B}=\left(u-u_{2 B \backslash B}\right)+(1-)\left(u-u_{2 B \backslash B}\right) .
$$

We say that a subset $A \subset \Omega$ is admissible if $A$ is open and $\partial A \cap \Omega$ is a smooth submanifold.

THEOREM 1. Under the assumptions of Lemma 1, each of the Sobolev type inequalities of Lemma 1 is equivalent to the following capacity type estimate:

(1) For an arbitrary ball $B \subset \subset \Omega$ there exists a constant $C_{1}=C(\Omega, B, f, g, p, q)$ such that

$$
\left(\int_{A} f(x) d x\right)^{p / q} \leqslant C_{1} \inf \int_{\Omega}|\nabla u(x)|^{p} g(x) d x
$$

for every admissible subset $A \subset \Omega$ with $A \cap B=\varnothing$. Here the infimum is taken over the set of all $u \in C^{\infty}(\Omega)$ that satisfy $\left.u\right|_{A} \geqslant 1$ and $\left.u\right|_{B}=0$.

Moreover, if $p=1$, then we have one more equivalent statement given as a weighted isoperimetric inequality.

(2) For any admissible subset $A \subset \Omega$ with $A \cap B=\varnothing$,

$$
\int_{A} f(x) d x \leqslant C_{2}\left(\int_{\partial A \cap \Omega} g d H^{n-1}\right)^{q} .
$$

Here $C_{2}=C(\Omega, B, f, g, q)$.

Remark 5. Letting $f=g=1$ and $q=n /(n-1)$, condition (2) above reduces to the usual isoperimetric inequality.

REMARK 6. The equivalence of the conditions from Lemma 1 with condition (1) above is the 'easy' part of the theorem. The 'hard' part is the equivalence with condition (2) for $p=1$.

Proof of Theorem 1. It suffices to prove the equivalence of the Theorem 1 conditions with condition (3) of Lemma 1. Denote this condition by Imb.

(1) $\Rightarrow \operatorname{Imb}$ : Let $u \in C^{\infty}(\Omega)$ satisfy $\left.u\right|_{B}=0$. The idea of the proof is as follows. We apply the capacity estimate to the level sets $A_{t}=\{|u| \geqslant t\}$. Since the $L^{q}$ norm of the function $u$ with respect to the measure given by $d \mu=f(x) d x$ can be recovered from the $\mu$ measures of the level sets $A_{t}$, we obtain the inequality Imb. Here are the details. 
Replacing $u$ by $|u|$, we can assume that $u \geqslant 0$. Let

$$
u_{k}=2^{-(k-1)} \min \left\{\max \left\{0, u-2^{k-1}\right\}, 2^{k-1}\right\}
$$

where $k \in \mathbb{Z}$. Then $u_{k}=1$ on $\left\{u \geqslant 2^{k}\right\}$, and

$$
\left|\nabla u_{k}\right|=\frac{1}{2^{\mathrm{k}-1}}|\nabla u| \chi_{\left\{2^{k-1} \leqslant u<2^{k}\right\}}
$$

almost everywhere. Hence

$$
\begin{aligned}
\int_{\Omega}|u|^{q} f d x & \approx \sum_{k=-\infty}^{\infty} 2^{k q} \int_{\left\{2^{k-1} \leqslant u<2^{k}\right\}} f d x \\
& \leqslant \sum_{k=-\infty}^{\infty} 2^{(k+1) q} \int_{\left\{u \geqslant 2^{k}\right\}} f d x \\
& \leqslant C \sum_{k=-\infty}^{\infty} 2^{k q}\left(\int_{\Omega}\left|\nabla u_{k}\right|^{p} g d x\right)^{q / p} \\
& \leqslant C \sum_{k=-\infty}^{\infty}\left(\int_{\left\{2^{k-1} \leqslant u<2^{k^{k}}\right\}}|\nabla u|^{p} g d x\right)^{q / p} \\
& \leqslant C\left(\sum_{k=-\infty}^{\infty} \int_{\left\{2^{k-1} \leqslant u<2^{k_{\}}}\right.}|\nabla u|^{p} g d x\right)^{q / p} \\
& =C\left(\int_{\Omega}|\nabla u|^{p} g d x\right)^{q / p} .
\end{aligned}
$$

In the last inequality, we have used the assumption that $q / p \geqslant 1$.

$\operatorname{Imb} \Rightarrow(1)$ : Let $u \in C^{\infty}(\Omega)$ satisfy $\left.u\right|_{A} \geqslant 1$ and $\left.u\right|_{B}=0$. We have

$$
\left(\int_{A} f d x\right)^{1 / q} \leqslant\left(\int_{\Omega}|u|^{q} f d x\right)^{1 / q} \leqslant C\left(\int_{\Omega}|\nabla u|^{p} g d x\right)^{1 / p} .
$$

Now let us assume that $p=1$. We shall prove that condition (2) is equivalent to Imb.

$(2) \Rightarrow \operatorname{Imb}$ : In the proof we need the following well known co-area formula $[11 ; 12 ; 33$, Theorem 1.2.4].

THEOREM 2. If $u$ is locally Lipschitz on $\Omega$, and $g$ is a nonnegative continuous function on $\Omega$, then

$$
\int_{\Omega}|\nabla u| g d x=\int_{0}^{\infty}\left(\int_{B_{t} \cap \Omega} g d H^{n-1}\right) d t
$$

where $B_{t}=\{x|| u(x) \mid=t\}$.

We follow the idea of Federer and Fleming [13] (cf. [51, Theorem 2.7.4]). Let $u \in C^{\infty}(\Omega)$ satisfy $\left.u\right|_{B}=0$. Replacing $u$ by $|u|$, we can assume that $u$ is nonnegative. Let $u_{t}=\min \{t, u\}$, where $t \geqslant 0$. Set $A_{t}=\{u \geqslant t\}$, and $B_{t}=\{u=t\}$. Obviously

for all $h \geqslant 0$. Hence

$$
u_{t+h} \leqslant u_{t}+h \chi_{A_{t}}
$$

$$
\left\|u_{t+h} f^{1 / q}\right\|_{q} \leqslant\left\|u_{t} f^{1 / q}\right\|_{q}+h\left(\int_{A_{t}} f\right)^{1 / q} \leqslant\left\|u_{t} f^{1 / q}\right\|_{q}+C h \int_{B_{t} \cap \Omega} g d H^{n-1} .
$$


This leads to the inequality

$$
\frac{d}{d t}\left\|u_{t} f^{1 / q}\right\|_{q} \leqslant C \int_{B_{t} \cap \Omega} g d H^{n-1} .
$$

Since the function $f$ is summable on $\Omega$, the function $\left\|u_{t} f^{1 / q}\right\|_{q}$ is differentiable almost everywhere with respect to $t$. Now, integrating both sides of (8) with respect to $t \in$ $(0, \infty)$ and applying the co-area formula, we obtain

$$
\begin{aligned}
\left(\int_{\Omega}|u|^{q} f d x\right)^{1 / q} & =\int_{0}^{\infty} \frac{d}{d t}\left\|u_{t} f^{1 / q}\right\|_{q} d t \\
& \leqslant C \int_{0}^{\infty}\left(\int_{B_{t} \cap \Omega} g d H^{n-1}\right) d t=C \int_{\Omega}|\nabla u| g d x .
\end{aligned}
$$

$\operatorname{Imb} \Rightarrow(2)$ : This implication is elegant but not very important, and we shall not use it in the sequel. Hence we only sketch the proof.

Lemma 2. Let $\Omega \subset \mathbb{R}^{n}$ be an arbitrary open set and $A \subset \Omega$ an admissible subset. Let $g: \Omega \rightarrow \mathbb{R}$ be a continuous and positive function with $\int_{\partial A \cap \Omega} g d H^{n-1}<\infty$. Then there exists a sequence $\left\{\omega_{m}\right\}_{m=1}^{\infty}$ of functions with the following properties:

(1) $\omega_{m} \in C^{\infty}(\Omega), 0 \leqslant \omega_{m} \leqslant 1$.

(2) $\omega_{m}=0$ in $\Omega \backslash A$.

(3) For every compact set $K \subset A$ there exists a number $m(K)$ such that $\left.\omega_{m}\right|_{K} \equiv 1$ for all $m \geqslant m(K)$.

(4)

$$
\lim _{m \rightarrow \infty} \int_{\Omega}\left|\nabla \omega_{m}\right| g d x=\int_{\partial A \cap \Omega} g d H^{n-1} .
$$

Lemma 2 is a generalisation of [33, Lemma 3.2.2]. The lemma follows by a standard modification of Maz'ya's argument. We skip the details.

Roughly speaking, Lemma 2 states that there exists a good, smooth approximation $\left\{\omega_{m}\right\}_{m=1}^{\infty}$ of the characteristic function of the set $A$.

Now it is easy to complete the proof of the implication that $\operatorname{Imb} \Rightarrow$ condition (2): simply apply the inequality stated in $\operatorname{Imb}$ to $u=\omega_{m}$, and let $m \rightarrow \infty$.

So far, we have dealt with inequalities for smooth functions. In order to extend our results to Sobolev spaces, we need a density result. In general, the existence of a weight may cause some serious problems (see [9]). However, our weights are sufficiently nice, and so the standard proof of density applies. For the sake of completeness, we supply some details.

Let $F=\left\{f_{\alpha\}_{|x|} \leqslant m}\right.$ be a family of positive and continuous functions on $\Omega$, and $P=\left\{p_{i}\right\}_{i=1}^{m}, 1 \leqslant p_{i}<\infty$, be a family of real numbers. We define the Sobolev space as follows:

$$
\begin{gathered}
W^{m, P}(\Omega, F)=\left\{u \in \mathscr{D}^{\prime}(\Omega) \mid D^{\alpha} u \in L^{p_{|\alpha|}}\left(\Omega, f_{\alpha}\right), \text { for }|\alpha| \leqslant m\right\} \\
\|u\|_{W^{m, P}(\Omega, F)}=\sum_{|\alpha| \leqslant m}\left(\int_{\Omega}\left|D^{\alpha} u\right|^{p_{|\alpha|}} f_{\alpha}(x) d x\right)^{1 / p_{|\alpha|}} .
\end{gathered}
$$

It is clear how to define $W_{\text {loc }}^{m, P}(\Omega, F)$. Moreover, $W_{0}^{m, P}(\Omega, F)$ stands for the closure of $C_{0}^{\infty}(\Omega)$ in the Sobolev norm. 
THEOREM 3. $W^{m, P}(\Omega, F)$ is a Banach space and $C^{\infty}(\Omega) \cap W^{m, P}(\Omega, F)$ is a dense subset. In fact, for every $u \in W_{\mathrm{loc}}^{m, P}(\Omega, F)$ and every $\varepsilon>0$, there exists a $g \in C^{\infty}(\Omega)$ with

(1) $u-g \in W_{0}^{m, P}(\Omega, F)$;

(2) $\|u-g\|_{W^{m, P}(\Omega, F)}<\varepsilon$.

The argument for the classical case is applied verbatim to establish that our Sobolev space is a Banach space. Proof of the second part of Theorem 3 requires only a minor modification to the usual proof of the Meyers-Serrin theorem, which is similar to that in [17]. The key observation which allows the standard arguments to work in the above weighted setting is that on every compact subdomain $\Omega^{\prime} \subset \subset \Omega$ the above weighted norm is equivalent to the unweighted one.

If one allows the weights to vanish even in a single point inside $\Omega$, then smooth functions can fail to be dense (see [9]).

Theorem 4 is an abstract version of the Rellich-Kondrachov compactness theorem.

THEOREM 4. Let $X$ be a set equipped with a finite measure $\mu$. Assume that a linear normed space $W$ of measurable functions on $X$ has the following two properties:

(1) There exists a $q>1$ such that the imbedding $W \subset L^{q}(X, \mu)$ is bounded.

(2) Every bounded sequence in $W$ contains a subsequence that converges almost everywhere.

Then the imbedding $W \subset L^{s}(X, \mu)$ is compact for every $1 \leqslant s<q$.

Remark 7. The assumption that $\mu(X)<\infty$ cannot be removed.

Proof of Theorem 4. Let $\left\{u_{j}\right\} \subset W$ be a bounded sequence; then it is also bounded in $L^{q}(X, \mu)$. Take a subsequence (still denoted by $\left\{u_{j}\right\}$ ) that converges almost everywhere. Now it suffices to prove that this sequence is a Cauchy sequence with respect to the $L^{s}(X, \mu)$ norm for each $s<q$. Fix $s<q$, and take an arbitrary small $\varepsilon>0$. According to Egoroff's theorem (here we use the assumption that $\mu(X)<\infty$ ), there exists a measurable subset $E \subset X$ such that $\mu(X \backslash E)<\varepsilon$ and $\left\{u_{j}\right\}$ converges uniformly on $E$. Now Theorem 4 follows from the estimate

$$
\begin{aligned}
\left(\int_{X}\left|u_{k}-u_{j}\right|^{s} d \mu\right)^{1 / s} \leqslant & \mu(X \backslash E)^{1-s / q}\left(\int_{X \backslash E}\left|u_{k}-u_{j}\right|^{q} d \mu\right)^{1 / q} \\
& +\left(\int_{E}\left|u_{k}-u_{j}\right|^{s} d \mu\right)^{1 / s} \\
\leqslant & C \varepsilon^{1-s / q}+\left(\int_{E}\left|u_{k}-u_{j}\right|^{s} d \mu\right)^{1 / s} \longrightarrow C \varepsilon^{1-s / q}
\end{aligned}
$$

as $k, j \rightarrow \infty$.

Theorem 4 leads to a general compact imbedding theorem for Sobolev spaces. First we need some notation.

Let $f$ and $g$ be two positive and continuous functions on $\Omega \subset \mathbb{R}^{n}$. By $L^{p}(\Omega, f)$, we denote the $L^{p}$ space with respect to the measure generated by $f(x) d x$, and by $W^{1, p}(\Omega, f ; g)$ we denote the completion of $C^{\infty}(\Omega)$ in the norm

$$
\|u\|_{L^{p}(\Omega, f)}+\|\nabla u\|_{L^{p}(\Omega, g)} .
$$


According to Theorem 3, this space coincides with the space defined via distributional derivatives. If $f \equiv g$, we simply write $W^{1, p}(\Omega, f)$, and in the unweighted case $f \equiv g \equiv 1$, we write $W^{1, p}(\Omega)$.

THEOREM 5. Let $W^{1, p}(\Omega, f ; g)$ be defined as above, and let $\mu$ be an arbitrary finite measure on $\Omega$ that is absolutely continuous with respect to the Lebesgue measure. Assume that $q>1$. If the imbedding $W^{1, p}(\Omega, f ; g) \subset L^{q}(\Omega, \mu)$ is bounded, then, for every $1 \leqslant s<q$, the imbedding $W^{1, p}(\Omega, f ; g) \subset L^{s}(\Omega, \mu)$ is compact.

REMARK 8. The assertion remains true if we also consider a slightly different Sobolev space obtained, for example, by replacing the norm $\|u\|_{L^{p}(\Omega, f)}$ in (9) by $\|u\|_{L^{1}\left(\Omega^{\prime}\right)}$, where $\Omega^{\prime} \subset \subset \Omega$.

COROLlary 1. If $f$ is as above, $\int_{\Omega} f(x) d x<\infty$, and $1 \leqslant q<p$, then the imbedding $W^{1, p}(\Omega, f) \subset L^{q}(\Omega, f)$ is compact.

Proof of Theorem 5. Let $\left\{u_{j}\right\} \subset W^{1, p}(\Omega, f ; g)$ be a bounded sequence. Since $f$ and $g$ are bounded away from 0 and $\infty$ on every compact subdomain $G \subset \subset \Omega$, we have $W^{1, p}(G, f ; g)=W^{1, p}(G)$ for each such $G$. Thus, requiring $G$ also to have smooth boundary, applying the classical Rellich-Kondrachov theorem to $W^{1, p}(G)$, and using the diagonal method, we can select from $\left\{u_{j}\right\}$ a subsequence that is convergent almost everywhere in $\Omega$. Now the assertion follows from Theorem 4 .

Now we can add one more equivalent condition to the list given in Lemma 1.

COROLlary 2. Let $f$ and $g$ be two positive and continuous functions on a domain $\Omega \subset \mathbb{R}^{n}$. Assume that $\int_{\Omega} f(x) d x<\infty$. Let $1 \leqslant p<q<\infty$. Then each of the conditions stated in Lemma 1 is equivalent to boundedness of the imbedding

$$
W^{1, p}(\Omega, f ; g) \subset L^{q}(\Omega, f) .
$$

Proof. Obviously, condition (2) of Lemma 1 implies (10). We prove the converse implication. The boundedness of the imbedding (10) means that

$$
\|u\|_{L^{q}(\Omega, f)} \leqslant C\left(\|u\|_{L^{p}(\Omega, f)}+\|\nabla u\|_{L^{p}(\Omega, g)}\right) .
$$

Now it suffices to prove that, for $u$ with $\int_{\Omega} u f=0$,

$$
\|u\|_{L^{p}(\Omega, f)} \leqslant C\|\nabla u\|_{L^{p}(\Omega, g)} .
$$

To this end, we use a classical argument which shows that compactness of imbedding implies Poincaré inequality (cf. [51, Lemma 4.1.3]). Suppose, contrary to our claim, that $\int_{\Omega} u_{k} f=0$ for $k=1,2, \ldots$ and

$$
1=\left\|u_{k}\right\|_{L^{p}(\Omega, f)}>k\|\nabla u\|_{L^{p}(\Omega, g)} .
$$

By Theorem 5, we can assume that $u_{k} \rightarrow u$ in $L^{p}(\Omega, f)$, and hence $\|u\|_{L^{p}(\Omega, f)}=1$ and $\int_{\Omega} u f=0$. On the other hand, $\nabla u_{k} \rightarrow 0$ in $L^{p}(\Omega, g)$. Since $\nabla u_{k} \rightarrow \nabla u$ in $\mathscr{D}^{\prime}(\Omega)$, we conclude that $\nabla u=0$, and so $u$ is constant ( $\Omega$ is connected). Thus $u=0$. The contradiction completes the proof.

We end this section with two elementary examples that illustrate Theorem 1 and Theorem 5. We give 'one line' proofs of the classical imbedding theorems in continuous domains, starshaped domains and in the $s$-cusps (definitions of these domains are given in the next section). 
Let $\Omega$ be a bounded continuous or starshaped domain, and let $B \subset \subset \Omega$ be a fixed ball. We shall shortly prove that the isoperimetric type inequality $|A| \leqslant$ $C H^{n-1}(\partial A \cap \Omega)$ holds for an arbitrary admissible subset $A \subset \Omega$ with $A \cap B=\varnothing$. According to Theorem 1, this inequality implies that

$$
\int_{\Omega}|u| d x \leqslant C \int_{\Omega}|\nabla u| d x
$$

for all $u \in C^{\infty}(\Omega)$ with $\left.u\right|_{B}=0$. If $1 \leqslant p<\infty$, then, applying (11) to $|u|^{p}$ and using the Hölder inequality and Lemma 1, we obtain the following result (a modification of this argument shows that (3) implies (4)).

COROLlaRy 3. If $\Omega$ is a bounded continuous or starshaped domain, and $1 \leqslant p<\infty$, then there exists a constant $C=C(p, \Omega)$ such that

$$
\left(\int_{\Omega}\left|u-u_{\Omega}\right|^{p} d x\right)^{1 / p} \leqslant C\left(\int_{\Omega}|\nabla u|^{p} d x\right)^{1 / p}
$$

whenever $u \in C^{\infty}(\Omega)$.

Proof. In order to complete the proof, we have to establish the isoperimetric inequality. Assume that $\Omega$ is a bounded and continuous domain (the case of starshaped domains is analogous). Since the question is local in nature, it suffices to consider the case when $A \subset \Omega$, and $\Omega$ is now an unbounded domain consisting of the points lying above the graph of a continuous function $f: \mathbb{R}^{n-1} \rightarrow \mathbb{R}$, and the diameter of the set $A$ is bounded from above. We have to establish the inequality $|A| \leqslant$ $C H^{n-1}(\partial A \cap \Omega)$. Let $\pi: \mathbb{R}^{n} \rightarrow \mathbb{R}^{n-1}$ be the orthogonal projection (onto the domain of definition of $f$ ). Then it is easy to see that $|A| \leqslant H^{n-1}(\pi(A)) \cdot \operatorname{diam} A \leqslant$ $H^{n-1}(\partial A \cap \Omega) \cdot \operatorname{diam} A$, as desired.

Another direct application is the following example, which is due to Maz'ya [28]. If $\Omega$ is a bounded s-cusp, $B \subset \subset \Omega$, and $A \subset \Omega$ with $A \cap B=\varnothing$ is an admissible subset, then it is not difficult to prove that the isoperimetric type inequality $|A| \leqslant$ $C H^{n-1}(\partial A \cap \Omega)^{q}$ holds for $q=1+1 /(s(n-1))$. Thus Theorem 1 and Theorem 5 lead to the following classical result.

COROLlary 4. If $\Omega$ is a bounded s-cusp, $s \geqslant 1$, and $1 \leqslant p<s(n-1)+1$, then there exists a constant $C=C(p, \Omega)$ such that

$$
\left(\int_{\Omega}\left|u-u_{\Omega}\right|^{q} d x\right)^{1 / q} \leqslant C\left(\int_{\Omega}|\nabla u|^{p} d x\right)^{1 / p}
$$

whenever $q=q_{0}=p(s(n-1)+1) /(s(n-1)+1-p)$ and $u \in C^{\infty}(\Omega)$. If $1 \leqslant q<q_{0}$, then the imbedding $W^{1, p}(\Omega) \subset L^{q}(\Omega)$ is compact.

The main results of this paper are Theorem 7, Theorem 8, Theorem 9 and Theorem 10. Three of these will be obtained as a consequence of Theorem 1 and Theorem 5.

\section{Domains}

One of the purposes of this paper is to establish imbedding theorems for certain classes of domains with irregular boundaries. In this section, we recall the definitions of these classes of domain. 
We say that the bounded domain $\Omega$ satisfies the cone condition if, for some fixed cone

$$
V=\left\{x_{1}^{2}+x_{2}^{2}+\ldots+x_{n-1}^{2} \leqslant C x_{n}^{2} \mid 0 \leqslant x_{n} \leqslant a\right\},
$$

every point $x \in \Omega$ is a vertex of a cone $V_{x} \subset \Omega$ congruent to $V$. For further properties of domains with the cone property, see [33]. We say that a bounded domain is continuous, Hölder continuous $\left(C^{0, \alpha}, 0<\alpha<1\right)$ or Lipschitz $\left(C^{0,1}\right)$ if, locally, its boundary is a graph of a continuous, Hölder continuous $\left(C^{0, \alpha}\right)$ or Lipschitz continuous function, respectively.

Of course, any Lipschitz domain satisfies the cone condition, but a general Hölder continuous domain does not satisfy it. The simplest nontrivial example of a Hölder continuous domain is a single $s$-cusp,$s \geqslant 1$. This is a domain defined by the inequality

$$
x_{1}^{2}+x_{2}^{2}+\ldots+x_{n-1}^{2} \leqslant C x_{n}^{2 s} \quad 0<x_{n}<a .
$$

If $s=1$, then we get a cone. In any $C^{0, \alpha}$ domain, each point of the boundary is a vertex of a $1 / \alpha$-cusp contained in $\Omega$.

We say that a bounded domain $\Omega$ is starshaped with respect to a point $x_{0}$ if, for every $x \in \Omega$, the interval $\overline{x x_{0}}$ is contained in $\Omega$. The domains starshaped with respect to a point are closely related to continuous domains. Namely, locally, by composition with an appropriate diffeomorphism, the boundary of a continuous domain can be mapped onto the boundary of a starshaped domain.

Now we define a class of much wilder domains. We say that a bounded domain $\Omega$ is $s$-John, $s \geqslant 1$, provided that there is a constant $C \geqslant 1$, and a distinguished point $x_{0} \in \Omega$ so that each point $x \in \Omega$ can be joined to $x_{0}$ (inside $\Omega$ ) by a rectifiable curve (called a John curve), $\gamma:[0, l] \rightarrow \Omega, \gamma(0)=x, \gamma(l)=x_{0}$, parameterised by arc length ( $l$ depends on $x$ ), and such that the distance to the boundary satisfies

$$
\operatorname{dist}(\gamma(t), \partial \Omega)>C^{-1} t^{s}
$$

for all $t \in[0, l]$. Note that $x_{0}$ can be replaced by any other point in $\Omega$. The constant $C$ in (12), however, depends on the choice of $x_{0}$.

If $s=1$, then we say, for simplicity, that $\Omega$ is a John domain and the condition (12) is called a 'twisted cone condition'. Note that a bounded domain that satisfies the cone condition is a John domain, whereas the cone condition can fail for a John domain. John domains were introduced by Martio and Sarvas [34]. They are named after F. John, who considered similar domains in [25]. They appear naturally in the context of holomorphic dynamical systems and quasiconformal mappings. Recently, Carleson, Jones and Yoccoz [8] characterised the polynomials for which the basin of infinity $A(\infty)$ is a John type domain. For further information concerning John domains, we refer the reader to $[35,37,48]$.

The $s$-John domains for $s>1$ are in general much wilder than the John domains, and hence many of the results that hold in 1-John domains do not extent to the case of $s$-John domains, $s>1$, which have not been as extensively studied as the usual John domains; see, however, $[\mathbf{5}, \mathbf{6}, \mathbf{4 1}]$. In the case in which $s>1$, condition (12) should perhaps be called a 'twisted cusp' or 'spire' condition. Buckley and the second author of this paper [6] have recently shown that a simply connected plane domain which supports a Sobolev-Poincaré inequality is an $s$-John domain for an appropriate $s$. 
In the sequel, we need the following result of Trotsenko [47].

THeORem 6. If $\Omega$ is a 1-John domain, then there exists $0<\delta<1$ such that

$$
\int_{\Omega} \operatorname{dist}(x, \partial \Omega)^{-\delta} d x<\infty .
$$

This theorem can also be easily deduced from [35, Lemma 2.8]. Note that, if $\Omega$ is a bounded domain with the cone property, then (13) holds for all $0<\delta<1$.

\section{Main results}

In this section, we use the notation $\varrho(x)=\operatorname{dist}(x, \partial \Omega)$, where $\Omega \subset \mathbb{R}^{n}$ is a fixed domain. Moreover, we denote by $u_{a}$ the average over $\Omega$ with respect to the measure induced by $d \mu(x)=\varrho(x)^{a} d x$, that is, $u_{a}=u_{\Omega}$. The main results of this section are Theorem 7, Theorem 8, Theorem 9 and Theorem 10. We remind the reader that, according to Theorem 5, the imbedding theorems that we establish below immediately result in associated compact imbeddings that we have chosen not to state for the sake of brevity.

Theorem 7. Let $\Omega \subset \mathbb{R}^{n}$ be an s-John domain, $s \geqslant 1$. Suppose that $a \geqslant 0$, $1 \leqslant q \leqslant n /(n-1)$, and $b=(a+n) /(s q)-n+1$. Then

$$
\left(\int_{\Omega}\left|u-u_{a}\right|^{q} \varrho(x)^{a} d x\right)^{1 / q} \leqslant C \int_{\Omega}|\nabla u| \varrho(x)^{b} d x
$$

for all $u \in C^{\infty}(\Omega)$.

Remark 9. It is immediate that $q$ cannot exceed the 'classical' exponent $n /(n-1)$.

In the particular case in which $a=b=0$, we have Corollary 5 .

CoROllary 5. If $\Omega \subset \mathbb{R}^{n}$ is an s-John domain and $1 \leqslant s \leqslant n /(n-1)$, then

$$
\left(\int_{\Omega}\left|u-u_{\Omega}\right|^{n /((n-1) s)} d x\right)^{(n-1) s / n} \leqslant C \int_{\Omega}|\nabla u| d x
$$

whenever $u \in C^{\infty}(\Omega)$.

Note that the exponent of integrability in Corollary 5 for $s=1$ is the same as for a bounded domain with a smooth boundary.

The standard method for showing that a certain imbedding theorem is sharp is to construct an appropriate 'rooms and corridors' domain. These domains are also called Nikodym's domains, after Nikodym [38], who was the first to consider a domain of this type as a counterexample to a Sobolev type imbedding. A number of Nikodym type domains have been constructed in [33]. 
Here we recall a version of Nikodym's domain. This example will show that Theorem 7 is sharp in the sense that one cannot obtain the inequality with better exponents under the assumptions of Theorem 7.

First we define a mushroom-like domain. A 'mushroom' $F$ of size $r$ consists of a cap $\mathscr{C}$, which is a ball of radius $r$, and an attached cylindrical stem $\mathscr{P}$ of height $r$ and with radius $r^{s}$. The cylinder is attached to the cap so as to create a mushroom shape.

A mushroom-like domain $\Omega$ consists of a cube $Q^{n}$ and an attached infinite sequence of mushrooms $F_{1}, F_{1}, \ldots$ growing on the 'top' of the cube. The mushrooms are disjoint, and the corresponding cylinders are perpendicular to the side of the cube that we have selected as the top of the cube. We can make the mushrooms pairwise disjoint if numbers $r_{i}$ associated with $F_{i}$ converge to 0 sufficiently fast as $i \rightarrow \infty$.

We shall not be very precise in our description as we believe that, once the reader understands the geometry and the basic idea, then the details become obvious. It is easy to see that the mushroom-like domain $\Omega$ is an $s$-John domain. Let $u_{i}$ be a piecewise linear function on $\Omega$ such that $u_{i}=0$ outside $F_{i}, u_{i}$ is equal to 1 on the cap $\mathscr{C}_{i}$, and $u_{i}$ is linear on the associated cylinder $\mathscr{P}_{i}$. Hence $\left|\nabla u_{i}\right|=r_{i}^{-1}$ on $\mathscr{P}$, and $\left|\nabla u_{i}\right|=0$ outside $\mathscr{P}$.

Assume that $1 \leqslant s \leqslant n /(n-1)$, and, for example, that one can prove Corollary 5 with the exponent $q>n /((n-1) s)$. Then, according to Lemma 1,

$$
\left(\int_{\Omega}\left|u_{i}\right|^{q} d x\right)^{1 / q} \leqslant C \int_{\Omega}\left|\nabla u_{i}\right| d x
$$

for a constant $C$ that does not depend on $i$. This leads to the inequality $r_{i}^{n / q} \leqslant C r_{i}^{s(n-1)}$ that cannot be true for sufficiently small $r_{i}$ (that is, for sufficiently large $i$ ).

Thus we have proved that the statement of Corollary 5 is sharp. It is easy to check that the same example proves the sharpness of the general case of Theorem 7 .

Applying inequality (4) to Corollary 5, we obtain

$$
\left(\int_{\Omega}\left|u-u_{\Omega}\right|^{n /(n-p(n-(n-1) s))} d x\right)^{(n-p(n-(n-1) s) /(n p)} \leqslant C\left(\int_{\Omega}|\nabla u|^{p} d x\right)^{1 / p}
$$

where $1 \leqslant s \leqslant n /(n-1)$ and $p<n /(n-(n-1) s)$.

In the case in which $s=1$ or $p=1$, this result is sharp. However, in the case when $s>1$ and $p>1$, the exponent on the left-hand side of (14) is not the best possible! Indeed, Corollary 6 provides the sharp exponent which exceeds that of (14) (see also the discussion after Corollary 6).

Before we proceed to the proof of Theorem 7, we state some lemmas that will be used in the sequel. The first lemma is the well known local isoperimetric inequality [16, Corollary $1.29 ; \mathbf{3 3}$, Lemma 1.2.1; 51, Theorem 5.4.3].

Lemma 3. If $B$ is an open ball of radius $r$ in $\mathbb{R}^{n}$, and $A$ is an admissible subset of $B$ with $|A \cap B| \leqslant|B| / 2$, then

$$
|A \cap B| \leqslant C_{1}(n) H^{n-1}(\partial A \cap B)^{n /(n-1)} .
$$

In particular, if $|A \cap B|=|B| / 2$, then

$$
r^{n} \leqslant C_{2}(n) H^{n-1}(\partial A \cap B)^{n /(n-1)} .
$$


We also need the following two well known covering lemmas. Lemma 4 is sometimes called a 'Vitali type covering lemma' [45; 51, Theorem 1.3.1].

Lemma 4. Let $E \subset \mathbb{R}^{n}$ be a bounded set, and let $E \subset \bigcup_{i \in I} B_{i}$, where $\left\{B_{i}\right\}_{i \in I}$ is a family of balls. Then we can select a subfamily (possibly finite) of pairwise disjoint balls $B_{1}, B_{2}, B_{3}, \ldots$ such that $E \subset \bigcup_{k=1}^{\infty} 5 B_{k}$.

Lemma 5 is due to Besicovitch $[\mathbf{2} ; \mathbf{3 3}$, Theorem $1.2 .1 ; \mathbf{3 6}$, p. $15 ; \mathbf{5 1}$, Theorem 1.3.5].

Lemma 5. Let $E \subset \mathbb{R}^{n}$ be a bounded set. Assume that we are given a family of balls $B_{x}=B(x, r(x))$ indexed by points in $E$. Then we can find a sequence (possibly finite) of points $x_{k} \in E, k=1,2,3, \ldots$ such that $E \subset \bigcup_{k=1}^{\infty} B_{x_{k}}$ and no point of $\mathbb{R}^{n}$ belongs to more than $C(n)$ balls.

Although Corollary 5 is an immediate consequence of Theorem 7, we prefer to give a direct proof of it. This is because the direct proof is much easier than that of Theorem 7, and also because the direct proof gives good insight into the ideas behind the proof of Theorem 7. We want to emphasise that the proof of Theorem 7 is independent from that of Corollary 5, and thus the reader might wish to skip the reading of the proof of Corollary 5.

Direct proof of Corollary 5. In this proof, we shall not use Lemma 5. Lemma 5 will be used in the proof of Theorem 7. Fix $x_{0} \in \Omega$. Let $B_{0}=B\left(x_{0}, \varrho\left(x_{0}\right) / 2\right)$. According to Theorem 1, it suffices to prove that, for an arbitrary admissible subset $A \subset \Omega$ with $A \cap B_{0}=\varnothing$, we have

$$
|A| \leqslant C H^{n-1}(\partial A \cap \Omega)^{n /((n-1) s)} .
$$

Let $x \in A$, and let $\gamma(t)$ be a John curve that joins $x$ with $x_{0}$, so that

$$
\operatorname{dist}(\gamma(t), \partial \Omega) \geqslant C_{1} t^{s}
$$

for all $0 \leqslant t \leqslant l$. Let $B_{t}=B\left(\gamma(t), C_{1} t^{s} / 2\right)$. For small $t$, this ball is contained in $A$, and when $\gamma(t)=x_{0}$, it is disjoint from $A$. Thus, if we trace along $\gamma,\left|B_{t} \cap A\right|=\left|B_{t}\right| / 2$ for some 'time' $t$. This means that there is a point $y$ on the John curve $\gamma$ and a radius $R_{y}$ with

(1) $\left|A \cap B\left(y, R_{y}\right)\right|=\left|B\left(y, R_{y}\right)\right| / 2$;

(2) $R_{y} \leqslant \varrho(y) / 2$;

(3) $x \in B\left(y, C R_{y}^{1 / s}\right)$.

The third condition follows easily from the fact that $t$ is the arc-length parameter of $\gamma$. Thus the family $\left\{B\left(y, C R_{y}^{1 / s}\right)\right\}_{y}$ forms a covering of $A$. According to the Vitali type lemma (Lemma 4), we can select a subfamily $\left\{B\left(y_{i}, C R_{y_{i}}^{1 / s}\right)\right\}_{i=1}^{\infty}$ of pairwise disjoint balls with

$$
A \subset \bigcup_{i=1}^{\infty} B\left(y_{i}, 5 C R_{y_{i}}^{1 / s}\right) .
$$


Hence, using Lemma 3, we conclude that

$$
\begin{aligned}
|A| & \leqslant C \sum_{i=1}^{\infty} R_{y_{i}}^{n / s} \leqslant C \sum_{i=1}^{\infty} H^{n-1}\left(\partial A \cap B\left(y_{i}, R_{y_{i}}\right)\right)^{n /((n-1) s)} \\
& \leqslant C\left(\sum_{i=1}^{\infty} H^{n-1}\left(\partial A \cap B\left(y_{i}, R_{y_{i}}\right)\right)\right)^{n /((n-1) s)} \leqslant C H^{n-1}(\partial A \cap \Omega)^{n /((n-1) s)} .
\end{aligned}
$$

In the second to last step, we used the assumption that $n /((n-1) s) \geqslant 1$. The proof is complete.

The above method could also be used to obtain some weighted inequalities, but it is not sufficient to cover the general statement of Theorem 7. The method would work (in the general case) if we knew that $R_{y} \approx \varrho(z)$ whenever $z \in B\left(y, R_{y}\right)$. Unfortunately, this is not true in general, and we only have the inequality $R_{y} \leqslant Q(z)$. This suggests that we should modify the argument as follows. We consider balls $B_{y}=B(y,(y) / 2)$, and we split $A$ into two parts: $A=\mathscr{G} \cup \mathscr{B}$. The good part, $\mathscr{G}$, consists (more or less) of these points $x$ for which $\left|B_{x}\right| \approx \varrho(x)^{n}$. The good part is essentially dealt with as above. To estimate the remaining 'bad' part, $\mathscr{B}$, we need a new argument. It turns out that the bad part can be rather easily dealt with by using the local isoperimetric inequality (Lemma 3 ).

Proof of Theorem 7. Let $x_{0}$ and $B_{0}$ be as above. According to Theorem 1, it suffices to prove that, for every admissible subset $A \subset \Omega$ with $A \cap B_{0}=\varnothing$, we have

$$
\int_{A}(x)^{a} d x \leqslant C\left(\int_{\partial A \cap \Omega}(x)^{b} d H^{n-1}\right)^{q} .
$$

For each $x \in \Omega$, we set $B_{x}=B\left(x, r_{x}\right)$, where $r_{x}=\varrho(x) / 2$. A trivial but useful observation is that, for $z \in B_{x}$, one has $\varrho(z) \approx r_{x}$. Let

$$
\begin{gathered}
\mathscr{G}=\left\{x \in A|| A \cap B_{x}\left|>\frac{1}{2}\right| B_{x} \mid\right\} \\
\mathscr{B}=A \backslash \mathscr{G} .
\end{gathered}
$$

( $\mathscr{B}$ corresponds to the 'bad' part of $\Omega$, and $\mathscr{G}$ to the 'good' part.) If $x \in \mathscr{G}$, then we trace along the John curve from $x$ to $x_{0}$, and pick a point $y$ on the curve with

$$
\left|A \cap B_{y}\right|=\frac{1}{2}\left|B_{y}\right| .
$$

From the $s$-John condition, we conclude that $|x-y| \leqslant C r_{y}^{1 / s}$, and hence that $x \in$ $B\left(y, C r_{y}^{1 / s}\right)$. Thus we obtain a covering of $\mathscr{G}$ by a family of balls $\left\{B\left(y, C r_{y}^{1 / s}\right)\right\}_{y}$ with points $y$ satisfying (15). Applying the Vitali type lemma (Lemma 4), we can select a sequence of pairwise disjoint balls $\left\{B_{y_{i}}\right\}_{i=1}^{\infty}=\left\{B\left(y_{i}, C r_{y_{i}}^{1 / s}\right\}_{i=1}^{\infty}\right.$ such that $\mathscr{G} \subset \bigcup_{i=1}^{\infty} B\left(y_{i}, 5 C r_{y_{i}}^{1 / s}\right)$. For the 'bad' part of $A$, we have $\mathscr{B} \subset \bigcup_{x \in \mathscr{B}} B_{x}$. Applying the covering lemma (Lemma 5), we find a collection $\left\{B_{x_{i}}\right\}_{i=1}^{\infty}$ such that $\mathscr{B} \subset \bigcup_{i=1}^{\infty} B_{x_{i}}$, and no point of $\Omega$ belongs to more than $C(n)$ of these balls. Now

$$
\int_{A}(x)^{a} d x=\int_{\mathscr{G}}(x)^{a} d x+\int_{\mathscr{B}}(a)^{a} d x .
$$


First we estimate the integral over the 'good' part. We have

$$
\int_{\mathscr{G}}(x)^{a} d x \leqslant \sum_{i=1}^{\infty} \int_{B\left(y_{i}, 5 C r_{y_{i}}^{1 / s}\right)}(x)^{a} d x
$$

Note that

$$
\int_{B\left(y_{i}, 5 C r_{y_{i}}^{1 / s}\right)} Q(x)^{a} d x \leqslant C\left(\int_{\partial A \cap B_{y_{i}}}(x)^{b} d H^{n-1}\right)^{q} .
$$

Indeed, since $\varrho(x) \leqslant C r_{y}^{1 / s}$ whenever $x \in B\left(y, C r_{y}^{1 / s}\right)$, the left-hand side can be estimated from above by $C r_{y_{i}}^{(a+n) / s}$. Using the fact that $Q(x) \approx r_{y_{i}}$ whenever $x \in B_{y_{i}}$ and Lemma 3, we see that the right-hand side is greater than or equal to $C\left(r_{y_{i}}^{b} r_{y_{i}}^{n-1}\right)^{q}=C r_{y_{i}}^{(a+n) / s}$, and thus (17) follows. Thus (16), (17) and the assumption $q \geqslant 1$ lead to

$$
\int_{\mathscr{G}}(x)^{a} d x \leqslant C\left(\sum_{i=1}^{\infty} \int_{\partial A \cap B_{y_{i}}}(x)^{b} d H^{n-1}\right)^{q} \leqslant C\left(\int_{\partial A \cap \Omega} Q(x)^{b} d H^{n-1}\right)^{q} .
$$

Now we estimate the 'bad' part using Lemma 3:

$$
\begin{aligned}
\int_{\mathscr{B}}\left((x)^{a} d x\right. & \leqslant \sum_{i=1}^{\infty} \int_{B_{x_{i}} \cap A}(x)^{a} d x \\
& \approx \sum_{i=1}^{\infty} r_{x_{i}}^{a}\left|B_{x_{i}} \cap A\right|^{1-q(n-1) / n}\left|B_{x_{i}} \cap A\right|^{q(n-1) / n} \\
& \leqslant C \sum_{i=1}^{\infty} r_{x_{i}}^{a+n-q(n-1)}\left(H^{n-1}\left(\partial A \cap B_{x_{i}}\right)^{q}\right. \\
& \leqslant C\left(\int_{\partial A \cap \Omega}(a)^{(a+n) / q-n+1} d H^{n-1}\right)^{q} \\
& \leqslant C\left(\int_{\partial A \cap \Omega}(x)^{b} d H^{n-1}\right)^{q} .
\end{aligned}
$$

Note that the assumption that $q \leqslant n /(n-1)$ was used for the estimate

$$
\left|B_{x_{i}} \cap A\right|^{1-q(n-1) / n} \leqslant C r_{x_{i}}^{n-q(n-1)} .
$$

Trotsenko's theorem (Theorem 6) allows for the following slight improvement of Theorem 7. Notice that the dimension of the boundary of a John domain is bounded away from $n$ by Trotsenko's theorem. However, given any $\varepsilon_{0}>0$, one can construct a John domain the boundary of which has dimension exceeding $n-\varepsilon_{0}$, and hence the bound $\varepsilon_{0}$ below can easily be checked to be essential.

THEOREM 8. Let $\Omega \subset \mathbb{R}^{n}$ be a 1-John domain. Then there exists $0<\varepsilon_{0}<1$ with the following property. If $1 \leqslant q \leqslant n /(n-1), 0<\varepsilon<\varepsilon_{0}$, and $b=(n-\varepsilon) / q-n+1$, then

$$
\left(\int_{\Omega}\left|u-u_{-\varepsilon}\right|^{q} \varrho^{-\varepsilon}(x) d x\right)^{1 / q} \leqslant C \int_{\Omega}|\nabla u| \varrho(x)^{b} d x
$$

for all $u \in C^{\infty}(\Omega)$. 
Proof. Note that the theorem that we proceed to prove is an extension of Theorem 7 to the case in which $-\varepsilon_{0}<a<0$ and $s=1$. In the proof of Theorem 7, the assumption that $a \geqslant 0$ was used only for the estimate

$$
\int_{B\left(y_{i}, 5 C r_{y_{i}}^{1 / s}\right)} Q(x)^{a} d x \leqslant C r_{y_{i}}^{(n+a) / s} .
$$

Hence the proof of Theorem 7 can be applied verbatim to give Theorem 8 , provided that we prove Lemma 6 .

Lemma 6. If $\Omega \subset \mathbb{R}^{n}$ is a 1-John domain, then there exist $0<\varepsilon_{0}<1$ and $C>0$ such that, for all $0<\varepsilon<\varepsilon_{0}$,

$$
\int_{B \cap \Omega}(x)^{-\varepsilon} d x \leqslant C r^{n-\varepsilon}
$$

whenever $B$ is a ball in $\mathbb{R}^{n}$ with radius $r$.

REMARK 10. If both $\Omega$ and $\overline{\mathbb{R}^{n}} \backslash \bar{\Omega}$ are 1-John domains (we identify $\overline{\mathbb{R}^{n}}$ with $S^{n}$ ), then Lemma 6 implies that, for certain $\varepsilon>0, \varrho^{\varepsilon} \in A_{1}$ (Muckenhoupt's class) (cf. [1, Lemma 2.1]). For example, if $\Omega=f(B)$, where $B$ is a ball and $f: \mathbb{R}^{n} \rightarrow \mathbb{R}^{n}$ is a quasiconformal mapping, then both $\Omega$ and $\overline{\mathbb{R}^{n}} \backslash \bar{\Omega}$ are 1-John domains.

Proof of Lemma 6. Let $B_{0}$ be as in the proof of Theorem 7, and let $r_{0}$ denote the radius of $B_{0}$. According to Theorem $7, \int_{\Omega}|v| d x \leqslant C \int_{\Omega}|\nabla v| \varrho d x$, whenever $\left.v\right|_{B_{0}}=0$. Let $|v|=|u| \varrho^{-\varepsilon}$, where the function $u \in C^{\infty}(\Omega)$ with $\left.u\right|_{B_{0}}=0$ is to be specified later. Using the fact that the Lipschitz constant of $\varrho$ is equal to 1, we obtain

$$
|\nabla v| \leqslant|\nabla u| \varrho^{\varepsilon}+\varepsilon|u| ß^{-(\varepsilon+1)}
$$

and hence

$$
\int_{\Omega}|u| \varrho^{-\varepsilon} \leqslant C \int_{\Omega}|\nabla u| \varrho^{-\varepsilon}+C \varepsilon \int_{\Omega}|u| \varrho^{-\varepsilon} .
$$

Pick $\varepsilon>0$ small enough so that $\int_{\Omega} \varrho^{-\varepsilon}<\infty$ and $C \varepsilon<1$; this is possible by Theorem 6. Assume that $u$ is bounded. Then (18) and $\int_{\Omega}|u| \varrho^{\varepsilon} d x<\infty$ imply that

$$
\int_{\Omega}|u| \varrho^{\varepsilon} \leqslant \frac{C}{1-C \varepsilon} \int_{\Omega}|\nabla u| \varrho^{-\varepsilon} \text {. }
$$

If $r \leqslant \operatorname{dist}(B, \partial \Omega)$ or $r$ is sufficiently large, say $r \geqslant r_{0} / 4$, then Lemma 6 follows easily. Hence we can assume that $r>\operatorname{dist}(B, \partial \Omega)$, and $r<r_{0} / 4$. Note that this implies that $2 B \cap B_{0}=\varnothing$. Let $u \in C^{\infty}(\Omega)$ be bounded and satisfy $u=1$ on $B \cap \Omega, u=0$ on $\Omega \backslash 2 B$, and $|\nabla u| \leqslant C / r$. Applying (19), we obtain

$$
\int_{B \cap \Omega} \varrho^{-\varepsilon} \leqslant \int_{\Omega}|u| \varrho^{-\varepsilon} \leqslant C \int_{2 B \cap \Omega} \frac{1}{r} \varrho^{-\varepsilon} \leqslant C r^{n-\varepsilon} .
$$


REMARK 11. The structure of the proof of Theorem 8 is somewhat 'funny'. We apply Trotsenko's theorem and Theorem 7 to obtain inequality (19), which is a special case of Theorem 8. Then we use the special case (19) to prove Lemma 6, and repeat the entire proof of Theorem 7 to establish the general case.

THEOREM 9. Let $\Omega \subset \mathbb{R}^{n}$ be an s-John domain, $s \geqslant 1$. Assume that the constants $a, b, p, q, s$ satisfy $a \geqslant 0,1 \leqslant p \leqslant q<(n+a) p /((n+b-1) s+1-p)$, and additionally $q \leqslant n p /(n-p)$, when $p<n$. Then

$$
\left(\int_{\Omega}\left|u-u_{a}\right|^{q}(\alpha)^{a} d x\right)^{1 / q} \leqslant C\left(\int_{\Omega}|\nabla u|^{p} Q(x)^{b} d x\right)^{1 / p}
$$

for all $u \in C^{\infty}(\Omega)$.

REMARK 12. It follows from the assumptions that $(n+b-1) s+1-p \geqslant 0$. In the case of equality, we allow $q$ to be any positive number.

By letting $a=b=0$, we have Corollary 6 . The case of Corollary 6 in which $p=q$ is due to Smith and Stegenga [41, Theorem 10].

Corollary 6. Let $\Omega \subset \mathbb{R}^{n}$ be an s-John domain, $s \geqslant 1$. If $1 \leqslant p \leqslant q<$ $n p /((n-1) s+1-p)$, then

$$
\left(\int_{\Omega}\left|u-u_{\Omega}\right|^{q} d x\right)^{1 / q} \leqslant C\left(\int_{\Omega}|\nabla u|^{p} d x\right)^{1 / p}
$$

whenever $u \in C^{\infty}(\Omega)$.

REMARK 13. If $(n-1) s+1-p \leqslant 0$, then the statement holds for any positive $q$.

We note that Corollary 6 can be proved directly using an argument that is considerably simpler than the proof of Theorem 9 (see Remark 16).

Let us compare Corollary 5 and Corollary 6.

If $p=1$, then $q<n /((n-1) s)$, and hence inequality (20) is weaker than that proved in Corollary 5. If $s=1$, then $q<n p /(n-p)$, and still Corollary 6 is not as good as Corollary 5, which leads to inequality (14). However, if $p>1$ and $s>1$, then inequality (20) is better than (14).

If $s>n /(n-1)$, then Corollary 5 does not apply at all, but Corollary 6 does. However, $p$ cannot be arbitrary. We need $p<n p /((n-1) s+1-p)$, or, equivalently, $p>(n-1)(s-1)$. The 'mushroom' example shows that this estimate is sharp, that is, that inequality (20) with $q \geqslant p$ does not hold when $1 \leqslant p<(n-1)(s-1)$. The fact that the Poincaré inequality (that is, (20) with $p=q$ ) holds for all sufficiently large $p$ is due to Smith and Stegenga [41, Theorem 10].

The mushroom example constructed above shows that, in the general case of Theorem 9, $q$ cannot exceed the given bound.

Before we proceed to the proof of Theorem 9, we prove the existence of an 'envelope extension operator' for 1-John domains. Although we shall not use this 
operator in the proof of Theorem 9, we think that such an 'intermission' will be convenient for the reader. Specifically, the proof of Theorem 9 generalises the arguments used in the construction of the envelope extension operator, and thus we hope that the construction presented below will help the reader to understand the proof of Theorem 9. First, we need to define the concept of an envelope extension operator.

Let $W(\Omega)$ be a normed space of measurable functions on $\Omega \subset \mathbb{R}^{n}$, and let $X$ be that for measurable functions on $\mathbb{R}^{n}$. We say that $\mathrm{T}: W(\Omega) \rightarrow X$ is an envelope extension operator if it is bounded and sublinear (that is, $|\mathrm{T}(u+v)| \leqslant|\mathrm{T} u|+|\mathrm{T} v|$ ), and, for any $u \in W(\Omega)$, the inequality

$$
|u(x)| \leqslant|\mathrm{T} u(x)|
$$

holds almost everywhere in $\Omega$ (the 'letter' $|u|$ is inside the 'envelope' $|\mathrm{T} u|$ ).

Obviously, for a general 1-John domain, there is no hope for a Sobolev extension operator. The simplest 1-John domain without the Sobolev extension property is a disk with a radius removed. However, as we shall see, any 1-John domain admits a Sobolev envelope extension operator.

TheORem 10. Let $\Omega \subset \mathbb{R}^{n}$ be a 1-John domain, and let $1<p<\infty$. Then there exists an envelope extension operator

$$
\mathrm{T}: W^{1, p}(\Omega) \longrightarrow W^{1, p}\left(\mathbb{R}^{n}\right) .
$$

Remark 14. Note that Theorem 10 cannot be trivial, as it can be shown to imply the Sobolev-Poincaré inequality for John domains.

Proof of Theorem 10. In the proof, we use a chaining argument similar to that used in $[18,19]$.

Let $x_{0} \in \Omega$ be a distinguished point, and let $B_{0}=B\left(x_{0},\left(x_{0}\right) / 4\right)$. In what follows, $x_{i}$ and $r_{i}$ denote the centre and the radius of a ball $B_{i}$, respectively.

We shall show that there is a constant $M$ that depends on the constant from the John condition (12) only and a constant $C$ that depends on $n$ only such that the following holds. For every $x \in \Omega \backslash 2 B_{0}$, there exists an infinite sequence (called a chain) of balls $B_{0}, B_{1}, \ldots$ with $2 B_{i} \subset \Omega$ that joins $x_{0}$ to $x$ in the following sense:

(1) $\left|B_{i} \cup B_{i+1}\right| \leqslant C\left|B_{i} \cap B_{i+1}\right|$.

(2) $\operatorname{dist}\left(x, B_{i}\right) \leqslant M r_{i}$, and $r_{i} \rightarrow 0, x_{i} \rightarrow x$, as $i \rightarrow \infty$.

(3) No point of $\Omega$ belongs to more than $C$ balls $B_{i}$.

There are plenty of papers that deal with various chaining techniques (cf. $[\mathbf{3}, \mathbf{4}, \mathbf{1 0}$, 14, 20-24, 27, 41, 42]), and the arguments needed to prove that the chain constructed below satisfies conditions (1), (2) and (3) immediately above are quite standard. Thus we shall be somewhat sketchy.

We cover $\gamma$ with balls as follows. Consider the collection of all balls

$$
B(y, \operatorname{dist}(y, \partial \Omega \cup\{x\}) / 4) \text { with } y \in \gamma .
$$

By the covering lemma (Lemma 5), we find the $B_{1}^{\prime}, B_{2}^{\prime}, \ldots$ from this collection that cover $\gamma \backslash\{x\}$ and have uniformly bounded overlap, depending only on $n$, at each point. 
Define $B_{i}=2 B_{i}^{\prime}$, and let $B_{0}$ be as above. Here $2 B_{i}^{\prime}$ is the ball with the same centre as $B_{i}^{\prime}$ but with twice the radius of $B_{i}^{\prime}$. Then the first part of property (2) holds for this sequence by the definition of $B_{i}$ because $\gamma$ is a John curve. Also, property (3) follows by volume comparison since the radii of $B_{i}$ and $B_{j}$ are comparable if $B_{i}$ and $B_{j}$ have nonempty intersection and the original balls $B_{i}^{\prime}$ have uniformly bounded overlap. Finally, property (1) and the second part of property (2) can easily be ensured by renumbering the balls as one traces along $\gamma$ from $x_{0}$ to $x$, and by disposing of the unnecessary balls.

Assume for a moment that $\left.u\right|_{B_{0}}=0$. According to the version of the Lebesgue differentiation theorem given in [45, Chapter 1, Section 1.8], for almost every $x \notin 2 B_{0}$ and the associated chain $\left\{B_{i}\right\}$, we have $u_{B_{i}} \rightarrow u(x)$, as $i \rightarrow \infty$, and hence

$$
\begin{aligned}
|u(x)| & \leqslant \sum_{i=0}^{\infty}\left|u_{B_{i}}-u_{B_{i+1}}\right| \\
& \leqslant \sum_{i=0}^{\infty}\left(\left|u_{B_{i}}-u_{B_{i} \cap B_{i+1}}\right|+\left|u_{B_{i+1}}-u_{B_{i} \cap B_{i+1}}\right|\right) \\
& \leqslant \sum_{i=0}^{\infty}\left(\oint_{B_{i} \cap B_{i+1}}\left|u-u_{B_{i}}\right|+\oint_{B_{i} \cap B_{i+1}}\left|u-u_{B_{i+1}}\right|\right) \\
& \leqslant C \sum_{i=0}^{\infty} \oint_{B_{i}}\left|u-u_{B_{i}}\right| \\
& \leqslant C \sum_{i=0}^{\infty} r_{i} \oint_{B_{i}}|\nabla u| .
\end{aligned}
$$

In the last step, we used the Poincare inequality. Note that, by condition (2), we have $|x-z| \leqslant C r_{i}$, for each $z \in B_{i}$, and hence

$$
|u(x)| \leqslant C \sum_{i=0}^{\infty} \int_{B_{i}} \frac{|\nabla u(z)|}{|x-z|^{n-1}} d z \leqslant C \int_{\Omega} \frac{|\nabla u(z)|}{|x-z|^{n-1}} d z .
$$

The constant $C$ does not depend on the choice of $x$. We have estimated $|u|$ by a Riesz potential which, according to Calderón-Zygmund theory, belongs to $W_{\text {loc }}^{1, p}\left(\mathbb{R}^{n}\right)$ as a function of the variable $x$ (cf. [45, Chapter 5, Section 2.3]). The remaining part of the proof is only of a cosmetic nature. Fix $\in C_{0}^{\infty}(\Omega),\left.\quad\right|_{2 B_{0}}=1$, and $\psi \in C_{0}^{\infty}\left(\mathbb{R}^{n}\right),\left.\psi\right|_{\Omega}=1$. Now we define the envelope extension operator by the formula

$$
T u(x)=\left(C \int_{\Omega} \frac{|\nabla(u-u)(z)|}{|x-z|^{n-1}} d z\right) \psi(x)+|(x) u(x)| .
$$

REMARK 15. A modification of the chaining technique used in the above proof easily extends to the setting of metric spaces [18, 19]. This technique is more elementary than the usual arguments based on the Boman chain condition and the Whitney decomposition, see $[\mathbf{1 4}, \mathbf{2 4}, \mathbf{2 7}]$. For the interested reader, let us point out that domains satisfying a Boman chain condition coincide with the 1-John domains even in a very abstract setting [7]. In the proof of Theorem 9, we shall also use a variant of the above chaining technique. 
REMARK 16. One can use the above method to obtain a rather simple proof of the unweighted case of Theorem 9, that is, Corollary 6. However, the weights of the general case cause serious difficulties, and we need to modify the chaining argument. This is the same phenomenon as that which we have already encountered in the proofs of Corollary 5 and Theorem 7.

Proof of Theorem 9. Let $B_{0}=B\left(x_{0}, Q\left(x_{0}\right) / 2\right)$, where $x_{0} \in \Omega$ is a distinguished point. It suffices to prove that there exists a constant $C>0$ such that, for every admissible subset $A \subset \Omega$ with $A \cap B_{0}=\varnothing$, we have

$$
\left(\int_{A}(x)^{a} d x\right)^{p / q} \leqslant C \int_{\Omega}|\nabla u|^{p}(x)^{b} d x
$$

whenever $u \in C^{\infty}(\Omega), u \geqslant 1$ on $A$, and $\left.u\right|_{B_{0}}=0$. For $z \in \Omega$, let $B_{z}=B\left(z, r_{z}\right)$, where $r_{z}=\varrho(z) / 2$. We set $B_{0}=B_{x_{0}}$.

Let $x \in A$ be arbitrary and let $\gamma$ be a John curve from $x$ to $x_{0}$. We trace along $\gamma$ from $x_{0}$ to $x$, and form a finite 'chain' of balls $B_{0}, B_{1}, \ldots, B_{k}(k$ depends on the choice of $x$ ) that joins $x_{0}$ to $x$ and has the following properties:

(i) Each of the balls $B_{i}$ is of the form $B_{z}$ with $z \in \gamma$. Moreover, $B_{0}=B_{x_{0}}$ is fixed, and $B_{k}=B_{x}$.

(ii) Consecutive balls are of comparable size and have considerable overlap. More precisely, we require that

for $i=0,1, \ldots, k-1$.

$$
\left|B_{i} \cup B_{i+1}\right| \leqslant M\left|B_{i} \cap B_{i+1}\right|
$$

(iii) For any $r>0$, the number of balls $B_{i}$ with radius $r_{i}>r$ is less than $M r^{(1-s) / s}$ when $s>1$, and less than $\left|\log _{2}\left(r^{-1} M \operatorname{diam} \Omega\right)\right|$ when $s=1$.

(iv) No point of $\Omega$ belongs to more than $M$ balls from $B_{0}, B_{1}, \ldots, B_{k}$.

The constant $M$ depends only on $n$ and the constant from the John condition (12). The required chain can easily be constructed by considering balls $B_{y}$ centred on $\gamma$ (see the proof of Theorem 10). The conditions (i), (ii) and (iv) immediately follow from the construction as in the proof of Theorem 10 . Thus we shall only prove property (iii), and, for simplicity, we only consider the case $s>1$, which is slightly harder than the case $s=1$.

Divide $\gamma$, starting from $x$, into pieces of the lengths $r^{1 / s},(2 r)^{1 / s}, \ldots,\left(2^{i} r\right)^{1 / s}, \ldots$ This is a finite sequence, of course. From properties (i) and (iv), we conclude that at most $C r^{1 / s} / r=C r^{(1-s) / s}$ balls with radius greater than $r$ correspond to the first piece of $\gamma$. Measuring in terms of arc length, the piece corresponding to $\left(2^{i} r\right)^{1 / s}$ is at least

$$
r^{1 / s}+(2 r)^{1 / s}+\ldots+\left(2^{\mathrm{i}-1} \mathrm{r}\right)^{1 / s} \approx\left(2^{i} r\right)^{1 / s}
$$

away from $x$, and so its distance to the boundary is at least $C 2^{i} r$. Hence it is covered by no more than $C\left(2^{i} r\right)^{(1-s) / s}$ balls from the chain. Thus the total number of balls with radius greater than $r$ does not exceed

$$
C\left(r^{(1-s) / s}+\sum_{i=1}^{\infty}\left(2^{i} r\right)^{(1-s) / s}\right) \approx r^{(1-s) / s}
$$


In order to estimate the integral over $A$, we divide $A$ as in the proof of Theorem 7 into the 'bad' and 'good' parts.

Let

$$
\begin{gathered}
\mathscr{G}=\left\{x \in A \mid u_{B_{x}} \geqslant \frac{1}{2}\right\} \\
\mathscr{B}=A \backslash \mathscr{G} .
\end{gathered}
$$

We have

$$
\int_{A}(x)^{a} d x=\int_{\mathscr{G}}(x)^{a} d x+\int_{\mathscr{B}}\left((x)^{a} d x .\right.
$$

We shall estimate these integrals separately. We begin with the integral over $\mathscr{G}$. Let $x \in \mathscr{G}$, and let $B_{0}, B_{1}, \ldots, B_{k}$ be a chain associated with $x$. Let $0<\lambda<1$. We estimate, using the triangle inequality, condition (ii), the Poincare inequality for $B_{i}$, and the Hölder inequality:

$$
\begin{aligned}
\frac{1}{2} & \leqslant\left|u_{B_{k}}-u_{B_{0}}\right| \leqslant \sum_{i=0}^{k-1}\left(\left|u_{B_{i}}-u_{B_{i} \cap B_{i+1}}\right|+\left|u_{B_{i+1}}-u_{B_{i} \cap B_{i+1}}\right|\right) \\
& \leqslant C \sum_{i=0}^{k} f_{B_{i}}\left|u-u_{B_{i}}\right| d y \\
& \leqslant C \sum_{i=0}^{k} r_{i} \int_{B_{i}}|\nabla u| d y \\
& \leqslant C\left(\sum_{i=0}^{k} r_{i}^{(1-\lambda) p /(p-1)}\right)^{(p-1) / p}\left(\sum_{i=0}^{k} r_{i}^{\lambda p-n} \int_{B_{i}}|\nabla u|^{p}\right)^{1 / p} .
\end{aligned}
$$

By an elementary scaling argument, we can assume that $\operatorname{diam} \Omega=1$. Fix small $\delta>0$, and set $\lambda=(s+p-1) /(s p)-\delta$. The number of $r_{i}$ with $2^{-(j+1)} \leqslant r_{i} \leqslant 2^{-j}$ does not exceed $C 2^{j(s-1) / s}$ (when $s>1$ ), and hence

$$
\sum_{i=0}^{k} r_{i}^{(1-\lambda) p /(p-1)} \leqslant C \sum_{j=0}^{\infty}\left(2^{-j}\right)^{(1-\lambda) p /(p-1)} 2^{j(s-1) / s}=C \sum_{j=0}^{\infty}\left(2^{-\delta p /(p-1)}\right)^{j}<C .
$$

Thus

$$
\left(\sum_{i=0}^{k} r_{i}^{(1-\lambda) p /(p-1)}\right)^{(p-1) / p} \leqslant C .
$$

It is easy to see that, also in the case $s=1$, expression (22) is finite (notice that then the $r_{i}$ decrease in a geometric fashion). Now (21) and (22) lead to

$$
\sum_{i=0}^{k} r_{i}^{\lambda p-n} \int_{B_{i}}|\nabla u|^{p} \geqslant C
$$

where the constant $C$ depends on $p, \delta, n$ and the constant from the $s$-John condition only. By the $s$-John condition $C r_{i} \geqslant|x-y|^{s}$, for $y \in B_{i}$, and since $\lambda p-n-b \leqslant 0$ (see Remark 12), we obtain

$$
r_{i}^{\lambda p-n-b} \leqslant C|x-y|^{s(\lambda p-n-b)}
$$

for $y \in B_{i}$. Note that $r_{i} \approx \varrho(y)$, when $y \in B_{i}$, and hence

$$
r_{i}^{\lambda p-n} \leqslant C Q(y)^{b}|x-y|^{s(\lambda p-n-b)} .
$$


For $y \in B_{i} \cap\left(2^{j+1} B_{k} \backslash 2^{j} B_{k}\right)$, we have $|x-y| \approx 2^{j} r_{k}$ (remember that $B_{k}$ is centred at $x$ ), and hence, for each such $y$,

$$
r_{i}^{\lambda p-n} \leqslant C\left(2^{j} r_{k}\right)^{s(\lambda p-n-b)}(y)^{b} .
$$

Moreover, since $r_{k}^{(\lambda p-n-b)} \geqslant 1$ (because $r_{k}<\operatorname{diam} \Omega=1$ ), and $s \geqslant 1$, we have, for $y \in B_{k}$,

$$
r_{k}^{\lambda p-n} \approx C r_{k}^{\lambda p-n-b} \varrho(y)^{b} \leqslant C r_{k}^{s(\lambda p-n-b)}(\alpha)^{b} .
$$

Thus (23)-(25), and the fact that no point of $\Omega$ belongs to more than $M$ of the balls $B_{i}$, lead to

$$
\begin{aligned}
C \leqslant & \sum_{i=0}^{k} r_{i}^{\lambda p-n} \int_{B_{i}}|\nabla u|^{p} \leqslant C r_{k}^{s(\lambda p-n-b)} \int_{B_{k}}|\nabla u|^{p} @(y)^{b} \\
& +C \sum_{j=0}^{\left|\log _{2} r_{k}\right|}\left(2^{j} r_{k}\right)^{s(\lambda p-n-b)} \int_{\left(2^{j+1} B_{k} \backslash 2^{j} B_{k}\right) \cap \Omega}|\nabla u|^{p} @(y)^{b} \\
\leqslant & C \sum_{l=0}^{\left|\log _{2} r_{k}\right|+1}\left(2^{l} r_{k}\right)^{s(\lambda p-n-b)} \int_{2^{l} B_{k} \cap \Omega}|\nabla u|^{p} \varrho(y)^{b} .
\end{aligned}
$$

Note that, above, it suffices to consider all $j$ with $j \leqslant\left|\log _{2} r_{k}\right|$ as we use the normalisation $\operatorname{diam} \Omega=1$. For any fixed $\varepsilon>0$, we have

$$
\sum_{l=0}^{\left|\log _{2} r_{k}\right|+1}\left(2^{l} r_{k}\right)^{\varepsilon}<r_{k}^{\varepsilon} \sum_{l=-\infty}^{\left|\log _{2} r_{k}\right|+1} 2^{l \varepsilon} \approx r_{k}^{\varepsilon} 2^{\left(\left|\log _{2} r_{k}\right|+1\right) \varepsilon}<C .
$$

Comparing (26) and (27), we see that the sum on the right-hand side of (26) exceeds the sum on the left-hand side of (27) (modulo a constant factor). Hence there exists an $l$ with

$$
\left(2^{l} r_{k}\right)^{\varepsilon}<C\left(2^{l} r_{k}\right)^{s(\lambda p-n-b)} \int_{2^{l} B_{k} \cap \Omega}|\nabla u|^{p} @(y)^{b} .
$$

In other words, there exists an $R_{x} \geqslant r_{x}=\varrho(x) / 2$ with

$$
\int_{\Omega \cap B\left(x, R_{x}\right)}|\nabla u|^{p} \varrho(y)^{b} d y \geqslant C R_{x}^{s(n+b-\lambda p)+\varepsilon} .
$$

Applying the Vitali type lemma (Lemma 4) to the covering $\left\{B\left(x, R_{x}\right)\right\}_{x \in \mathscr{G}}$ of the set $\mathscr{G}$, we can select pairwise disjoint balls $B_{(1)}, B_{(2)}, B_{(3)}, \ldots$ such that $\mathscr{G} \subset \bigcup_{i=1}^{\infty} 5 B_{(i)}$. Let $r_{(i)}$ denote the radius of the ball $B_{(i)}$. Set $\delta=\varepsilon /(s p)$. Then

$$
s(n+b-\lambda p)+\varepsilon=(n+b-1) s+1-p+2 \varepsilon .
$$

For $y \in B\left(x, R_{x}\right)$, we have $\varrho(y) \leqslant 3 R_{x}$, and hence $\varrho(y)^{a} \leqslant C R_{x}^{a}$ (here we use the assumption $a \geqslant 0$ ). Now we are ready to estimate the integral over $\mathscr{G}$ :

$$
\begin{aligned}
\int_{\mathscr{G}}(a)^{a} d x & \leqslant \sum_{i=1}^{\infty} \int_{5 B_{(i)} \cap \Omega}(x)^{a} d x \\
& \leqslant C \sum_{i=1}^{\infty} r_{(i)}^{a+n} \\
& \left.\leqslant C \sum_{i=1}^{\infty}\left(\int_{\Omega \cap B_{(i)}}|\nabla u|^{p} \underline{(} y\right)^{b} d y\right)^{(a+n) /((n+b-1) s+1-p+2 \varepsilon)} \\
& \leqslant C\left(\sum_{i=1}^{\infty} \int_{\Omega \cap B_{(i)}}|\nabla u|^{p} \underline{Q}(y)^{b} d y\right)^{(a+n) /((n+b-1) s+1-p+2 \varepsilon)} .
\end{aligned}
$$


In the third inequality, we applied (28) and (29), and, in the second to last step, we used the inequality

$$
\frac{a+n}{(n+b-1) s+1-p+2 \varepsilon} \geqslant 1
$$

which holds for sufficiently small $\varepsilon$. Now it remains to estimate the integral over $\mathscr{B}$.

We have $\mathscr{B} \subset \bigcup_{x \in \mathscr{B}} B_{x}$. Let $\left\{B_{x_{i}}\right\}_{i=1}^{\infty}$ be a subcovering as in the Besicovitch lemma (Lemma 5). Since $u \geqslant 1$ on $A$, and $u_{B_{x_{i}}} \leqslant 1 / 2$, we obtain

$$
\left|u(y)-u_{B_{x_{i}}}\right|^{q} \geqslant 2^{-q}
$$

for $y \in A \cap B_{x_{i}}$. Hence, using the Sobolev-Poincaré inequality, we get

$$
\left|A \cap B_{x_{i}}\right| \leqslant C \int_{A \cap B_{x_{i}}}\left|u-u_{B_{x_{i}}}\right|^{q} \leqslant C r_{x_{i}}^{(n / q-n / p+1) q}\left(\int_{B_{x_{i}}}|\nabla u|^{p}\right)^{q / p} .
$$

In the last inequality, we used the assumption that $q \leqslant n p /(n-p)$, when $p<n$. Now we have

$$
\begin{aligned}
& \int_{\mathscr{B}}(y)^{a} d y \leqslant \sum_{i=1}^{\infty} \int_{B_{x_{i}} \cap A}(y)^{a} d y \approx \sum_{i=1}^{\infty} r_{x_{i}}^{a}\left|B_{x_{i}} \cap A\right| \\
& \leqslant C \sum_{i=1}^{\infty} r_{x_{i}}^{a+(n / q-n / p+1) q}\left(\int_{B_{x_{i}}}|\nabla u|^{p}\right)^{q / p} \\
& \leqslant C\left(\int_{\Omega}|\nabla u|^{p} \varrho^{(n+a) / q-n / p+1) p} d x\right)^{q / p} .
\end{aligned}
$$

Theorem 9 follows since $((n+a) / q-n / p+1) p \geqslant b$.

Acknowledgements. We would like to thank Agnieszka Kałamajska for an argument that simplified our original proof of Theorem 5 .

The research in the paper was begun while the first author was visiting the University of Helsinki and the University of Jyväskylä in 1994, and completed during his stay at the ICTP in Trieste in 1995. He wishes to thank the departments and the ICTP for their hospitality.

Note added in proof. Recently, T. Kilpeläinen and J. Malý have proved that the inequality of Theorem 9 holds with the borderline exponent

$$
q=(n+a) p /((n+b-1) s+1-p) .
$$

\section{References}

1. K. Astala, 'Calderón's problem for Lipschitz classes and the dimension of quasicircles', Rev. Mat. Iberoamericana 4 (1988) 469-486.

2. A. S. Besicovitch, 'A general form of the covering principle and relative differentiation of additive functions', Proc. Cambridge Philos. Soc. 41 (1945) 103-110.

3. B. BojARSKI, 'Remarks on Sobolev imbedding inequalities', Proceedings of the Conference on Complex Analysis, Joensu 1987, Lecture Notes in Mathematics 1351 (Springer 1988).

4. J. Boman, ' $L^{p}$-estimates for very strongly elliptic systems', Report 29, Department of Mathematics, University of Stockholm, 1982.

5. S. M. Buckley and P. Koskela, 'Sobolev-Poincaré inequalities for $p<1$ ', Indiana Univ. Math. J. 43 (1994) 221-240.

6. S. M. Buckley and P. Koskela, 'Sobolev-Poincaré implies John', Math. Res. Lett. 2 (1995) 577-593.

7. S. Buckley, P. Koskela and G. Lu, 'Boman equals John', Proceedings of the XVI Rolf Nevanlinna Colloquium (Jaensuu, 1995) (de Gruyter, Berlin, 1996) 91-99. 
8. L. Carleson, P. W. Jones and J. C. Yoccoz, 'Julia and John', Bol. Soc. Brasil. Mat. 25 (1994) 1-30.

9. V. Chiadò Piat and F. Serra Cassano, 'Some remarks about the density of smooth functions in weighted Sobolev spaces', J. Convex Analysis 1 (1994) 135-142.

10. S. K. ChuA, 'Weighted Sobolev inequalities on domains satisfying the chain condition', Proc. Amer. Math. Soc. 117 (1993) 449-457.

11. H. Federer, 'Curvature measures', Trans. Amer. Math. Soc. 93 (1959) 418-491.

12. H. FEDERER, Geometric measure theory (Springer, 1969).

13. H. Federer and W. H. Fleming, 'Normal and integral currents', Ann. Math. 72 (1960) 458-520.

14. B. Franchi, C. E. Gutiérrez and R. L. Wheeden, 'Weighted Sobolev-Poincaré inequalities for Grushin type operators', Comm. Partial Differential Equations 19 (1994) 523-604.

15. E. Gagliardo, 'Proprieta di alcune classi di funzioni in piu variabli', Richerche Mat. 7 (1958) $102-137$.

16. E. Giusti, Minimal surfaces and functions of bounded variation (Birkhäuser, Boston, 1984).

17. P. HaJtasz, 'Note on Meyers-Serrin's theorem', Expo. Math. 11 (1993) 377-379.

18. P. Hajlasz and P. Koskela, 'Sobolev meets Poincaré', C.R. Acad. Sci. Paris 320 (1995) 1211-1215.

19. P. Hajlasz and P. Koskela, 'Sobolev met Poincaré, preprint.

20. R. Hurri, 'Poincaré domains in $\mathbb{R}^{n}$ ', Ann. Acad. Sci. Fenn. Ser. A I Math. Dissertationes 71 (1988) $1-42$.

21. R. Hurri, 'The weighted Poincaré inequality', Math. Scand. 67 (1990) 145-160.

22. R. Hurri-Syruänen, 'An improved Poincaré inequality', Proc. Amer. Math. Soc. 120 (1994) 213-232.

23. T. IwANIEC and C. A. NOLDER, 'Hardy-Littlewood inequality for quasiregular mappings in certain domains in $\mathbb{R}^{n}$, Ann. Acad. Sci. Fenn. Ser. A I Math. 10 (1985) 267-282.

24. D. JERISON, 'The Poincaré inequality for vector fields satisfying Hörmander's condition', Duke Math. J. 53 (1986) 503-523.

25. F. John, 'Rotation and strain', Comm. Pure Appl. Math. 4 (1961) 391-414.

26. V. A. Kaimanovich, 'Dirichlet norms, capacities and generalized isoperimetric inequalities for Markov operators', Potential Anal. 1 (1992) 61-82.

27. G. Lu, 'The sharp Poincaré inequality for free vector fields: an endpoint result', Rev. Mat. Iberoamericana 10 (1994) 453-466.

28. V. G. MAZ'YA, 'Classes of domains and imbedding theorems for function spaces', Dokl. Akad. Nauk SSSR 133 (1960) 527-530 (in Russian); Soviet Math. Dokl. 1 (1960) 882-885 (in English).

29. V. G. MAZ'YA, 'The $p$-conductivity and theorems on imbedding certain functions spaces into a $C$ space', Dokl. Akad. Nauk SSSR 140 (1961) 299-302 (in Russian); Soviet Math. Dokl. 2 (1961) 1200-1203 (in English).

30. V. G. MaZ'ya, 'Classes of sets and measures connected with imbedding theorems', Imbedding theorems and their applications, Proceedings Symposium Baku 1966 (Nauka, Moscow, 1970) $142-159$ (in Russian).

31. V. G. Maz'ya, 'On certain integral inequalities for functions of many variables', Problems of mathematical analysis 3 (Leningrad University, Leningrad, 1972) 33-68 (in Russian); J. Soviet Math. 1 (1973) 205-234 (in English).

32. V. G. MaZ'ya, 'On the summability of functions in S. L. Sobolev spaces', Problems of mathematical analysis 5 (Leningrad University, Leningrad, 1975) 66-98 (in Russian).

33. V. G. Maz'ya, Sobolev spaces (Springer, 1985).

34. O. Martio and J. Sarvas, 'Injectivity theorems in plane and space', Ann. Acad. Sci. Fenn. Ser. A I Math. $4(1978 / 79)$ 383-401.

35. O. Martio and M. Vuorinen, 'Whitney cubes, p-capacity, and Minkowski content', Expo. Math. 5 (1987) 17-40.

36. F. Morgan, Geometric measure theory (Academic Press, 1988).

37. R. NÄKKI and J. VÄIsÄLÄ, 'John discs', Expo. Math. 9 (1991) 3-43.

38. O. Nikodyм, 'Sur une classe de fonctions considérémes le problée de Dirichlet', Fund. Math. 21 (1933) $129-150$.

39. L. Nirenberg, 'On elliptic differential equations', Ann. Scuola Norm. Pisa (III) 13 (1959) 1-48.

40. B. OpIC and A. KufNer, Hardy-type inequalities, Research Notes in Mathematics 219 (Pitman, 1990).

41. W. Smith and D. A. Stegenga, 'Hölder domains and Poincaré domains', Trans. Amer. Math. Soc. 319 (1990) 67-100.

42. W. Smith and D. A. Stegenga, 'Sobolev imbeddings and integrability of harmonic functions in Hölder domains', Potential theory (de Gruyter, Berlin, 1992).

43. S. L. Sobolev, 'On the estimates relating to families of functions having derivatives that are square integrable', Dokl. Akad. Nauk SSSR 1 (1936) 267-270 (in Russian).

44. S. L. Sobolev, 'On a theorem of functional analysis', Mat. Sb. 46 (1938) 471-497 (in Russian); Amer. Math. Soc. Transl. Ser. 234 (1963) 39-68 (in English).

45. E. Stein, Singular integrals and differentiability properties of functions (Princeton University Press, 1970).

46. E. W. Stredulinsky, Weighted inequalities and degenerate elliptic partial differential equations, Lecture Notes in Mathematics 1074 (Springer, 1984). 
47. D. A. Trotsenko, 'Properties of regions with nonsmooth boundary', Sibirsk. Mat. Zh. 22 (1981) 221-224 (in Russian).

48. J. VÄIsÄLÄ, 'Uniform domains', Tohoku Math. J. 40 (1988) 101-118.

49. T. VARopoulos, 'Isoperimetric inequalities and Markov chains', J. Funct. Anal. 63 (1985) 215-239.

50. T. Varopoulos, L. Saloff-Coste and T. Coulhon, Analysis and geometry on groups (Cambridge University Press, 1992).

51. W. Ziemer, Weakly differentiable functions, Graduate Texts in Mathematics 120 (Springer, 1989).

Instytut Matematyki

Uniwersytet Warszawski

Banacha 2

02-097 Warszawa

Poland

E-mail: hajlasz@mimuw.edu.pl
Department of Mathematics University of Michigan

Ann Arbor

MI 48109

USA

Present address:

Department of Mathematics

University of Jyväskylä

PO Box 35

Fin-40351 Jyväskylä

Finland

E-mail: pkoskela@math.jyu.fi 